EPJ Web of Conferences 13, 02003 (2011)

DOI: $10.1051 /$ epjconf/20111302003

C Owned by the authors, published by EDP Sciences, 2011

\title{
The effect of the Polyakov loop on the chiral phase transition
}

\author{
G. Markó ${ }^{1, a}$ and Zs. Szép ${ }^{2, b}$ \\ 1 Department of Atomic Physics, Eötvös University, H-1117 Budapest, Hungary \\ 2 Centre de Physique Théorique, Ecole Polytechnique, CNRS, 91128 Palaiseau Cedex, France
}

\begin{abstract}
The Polyakov loop is included in the $S U(2)_{L} \times S U(2)_{R}$ chiral quark-meson model by considering the propagation of the constituent quarks, coupled to the $(\sigma, \pi)$ meson multiplet, on the homogeneous background of a temporal gauge field, diagonal in color space. The model is solved at finite temperature and quark baryon chemical potential both in the chiral limit and for the physical value of the pion mass by using an expansion in the number of flavors $N_{f}$. Keeping the fermion propagator at its tree-level, a resummation on the pion propagator is constructed which resums infinitely many orders in $1 / N_{f}$, where $O\left(1 / N_{f}\right)$ represents the order at which the fermions start to contribute in the pion propagator. The influence of the Polyakov loop on the tricritical or the critical point in the $\mu_{q}-T$ phase diagram is studied for various forms of the Polyakov loop potential.
\end{abstract}

\section{Introduction}

The low-energy effective models of the QCD, such as the Nambu-Jona-Lasinio (NJL) model and the chiral quarkmeson model (QM), are based on the global chiral symmetry of the QCD. They are very useful to qualitatively understand many aspects related to the spontaneous breaking of the chiral symmetry and its restoration at finite temperature and density. However, the absence of gluonic effective degrees of freedom and the lack of color clustering alter the reliability of the quantitative thermodynamic predictions of these models, such as the equation of state or the location of the critical end point (CEP) in the $\mu_{q}-T$ phase diagram.

Some information on the quark confinement can be incorporated in the effective models through an effective degree of freedom, the Polyakov loop, which is a good order parameter for the deconfinement phase transition in the absence of dynamical quarks. The coupling of the Polyakov loop to the chiral effective models mimics the effect of confinement by statistically suppressing at low temperature the contribution of one- and two-quark states relative to the three-quark states. This feature makes the Polyakovloop extended effective models more appropriate for the description of the low-temperature phase and for quantitative comparison with the thermodynamic observables on the lattice [1-3]. Better agreement is expected up to $T \simeq(1.5-2) T_{c}$ above which the transverse gluonic degrees of freedom dominate in thermodynamic quantities, such as the pressure, over the longitudinal ones represented by the Polyakov loop.

\footnotetext{
a e-mail: smarkovics@hotmail.com

b e-mail: szepzs@achilles.elte.hu. Speaker. On leave from Statistical and Biological Physics Research Group of the Hungarian Academy of Sciences, H-1117 Budapest, Hungary.
}

Some solutions of the Polyakov loop extended quarkmeson model (PQM) appearing in the literature completely disregard quantum effects. The effect of including the quantum fluctuation in the PQM model was recently studied in [4-6] using functional renormalization group methods and also in [7], where it was shown that the inclusion of the fluctuations has a significant effect on the location of the CEP, which is pushed to higher values of $\mu_{q}$. In this contribution we review the results on the $\mu_{q}-T$ phase diagram obtained in [7] as a result of including different forms of the effective Polyakov loop potential, as compared to those previously obtained in [8] in the chiral limit of the two flavor QM using the resummation of the perturbative series provided by the large- $N_{f}$ approximation. Starting with the $\Phi$-derivable formalism, the approximations done to parametrize and solve the model are also discussed.

\section{The model in the large- $N_{f}$ approximation}

The ingredients for the PQM model are the $(\sigma, \pi)$ meson multiplet and the constituent quark fields $\psi$ coupled to them. These latter propagate on the homogeneous background of a temporal gauge field. In order to be able to use a large$N_{f}$ expansion we consider $N_{f}$ constituent quarks and correspondingly $N-1$ pions $\left(\sqrt{N}=N_{f}\right)$. Performing some rescaling with $N_{f}$ (see $[7,8]$ for details), which assures the finiteness of the tree-level constituent quark mass $m_{q}=g v$ as $N_{f} \rightarrow \infty$, the Lagrangian of the model reads after separating the vacuum expectation value $v N_{f}$ of the $\sigma$ field as :

$$
\begin{aligned}
\mathcal{L} & =-N\left[\frac{\lambda}{24} v^{4}+\frac{1}{2} m^{2} v^{2}-h v\right]-\sqrt{N}\left[\frac{\lambda}{6} v^{3}+m^{2} v-h\right] \sigma \\
& +\frac{1}{2}\left[(\partial \sigma)^{2}+(\partial \pi)^{2}\right]-\frac{1}{2} m_{\sigma 0}^{2} \sigma^{2}-\frac{1}{2} m_{\pi 0}^{2} \pi^{2}
\end{aligned}
$$




$$
\begin{aligned}
& -\frac{\lambda v}{6 \sqrt{N}} \sigma \rho^{2}-\frac{\lambda}{24 N} \rho^{4}+\bar{\psi}\left[\left(i \partial^{\mu}+\delta^{\mu 0} A_{0}\right) \gamma_{\mu}-m_{q}\right] \psi \\
& -\frac{g}{\sqrt{N}}\left[\bar{\psi}\left(\sigma+i \sqrt{2 N_{f}} \gamma_{5} T^{a} \pi^{a}\right)\right] \psi
\end{aligned}
$$

where $\rho^{2}=\sigma^{2}+\pi^{2}$ and the tree-level sigma and pion masses are $m_{\sigma 0}^{2}=m^{2}+\lambda v^{2} / 2$ and $m_{\pi 0}^{2}=m^{2}+\lambda v^{2} / 6 . A_{0}$ is the temporal component of the gauge field, taken to be constant in the mean-field approximation.

\subsection{The grand potential in the $\Phi$-derivable approximation}

At finite temperature $T=1 / \beta$, after analytical continuation to imaginary time $t \rightarrow i \tau, A_{0} \rightarrow i A_{4}$, the grand partition function $Z$ and the grand potential $\Omega\left(T, \mu_{B}\right)$ of the spatially uniform system defined by (1) are defined as

$$
Z=\operatorname{tr}\left\{\exp \left[-\beta\left(H_{0}\left(A_{4}\right)+H_{\text {int }}-\mu_{B} Q_{B}\right)\right]\right\}=e^{-\beta \Omega},
$$

where $\mu_{B}$ is the baryon chemical potential. $H_{\mathrm{int}}$ is the interacting part of the Hamiltonian constructed from (1). The $A_{4}$-dependent free Hamiltonian $H_{0}\left(A_{4}\right)$ for two quark flavors $u$ and $d$ reads

$$
H_{0}\left(A_{4}\right)=H_{0}+\int d^{3} x\left[i u_{i}^{\dagger}(x) A_{4}^{i j} u_{j}(x)+i d_{i}^{\dagger}(x) A_{4}^{i j} d_{j}(x)\right]
$$

where $H_{0}$ is the quadratic part of the Hamiltonian at vanishing $A_{4}$ and $i, j$ denotes color indices. In the so-called Polyakov gauge $A_{4}$ is diagonal in color space, that is $A_{4}=$ $\operatorname{diag}\left(\phi_{+}, \phi_{-},-\left(\phi_{+}+\phi_{-}\right)\right)$. The conserved baryon charge $Q_{B}$ appearing in (2) can be expressed in terms of the particle number operators as $Q_{B}=\frac{1}{3} \sum_{i=1}^{3} N_{q, i}$, with $N_{\mathrm{q}, \mathrm{i}}=N_{u, i}+$ $N_{d, i}-N_{\bar{u}, i}-N_{\bar{d}, i}$ and e.g. $N_{u, i}=\int d^{3} x\left(u_{i}^{\dagger} u_{i}+d_{i}^{\dagger} d_{i}\right)$. Then, combining $H_{0}\left(A_{4}\right)$ and $\mu_{B} Q_{B}$, the effect of fermions propagating on the constant background $A_{4}$, diagonal in the color space is like having imaginary chemical potential for color. Following Ref. [9], one introduces color-dependent chemical potential for fermions

$$
\mu_{1,2}=\mu_{q}-i \phi_{ \pm}, \quad \mu_{3}=\mu_{q}+i\left(\phi_{+}+\phi_{-}\right),
$$

where $\mu_{q}=\mu_{B} / 3$ is the quark baryon chemical potential. Then, introducing the notation $\mathcal{H}=H_{0}-\sum_{i=1}^{3} \mu_{i} N_{q, i}$, one can write $Z$ as a path integral over the fields, generically denoted by $\Psi$

$$
Z=e^{-\beta \Omega_{0}} \frac{\int[\mathcal{D} \Psi]\left\{e^{-\beta \mathcal{H}} \mathcal{P} \exp \left[-\int_{0}^{\beta} d \tau H_{\text {int }}(\tau)\right]\right\}}{\int[\mathcal{D} \Psi] e^{-\beta \mathcal{H}}}
$$

where $\Omega_{0}$ is the grand potential of the unperturbed system with fermions having color-dependent chemical potential

$$
e^{-\beta \Omega_{0}}=\int[\mathcal{D} \Psi] e^{-\beta \mathcal{H}}
$$

In the $\Phi$-derivable approximation of Ref. [10], which is also called two-particle irreducible (2PI) approximation, the grand potential $\Omega \equiv \Omega\left[G_{\pi}, G_{\sigma}, G, v, \Phi, \bar{\Phi}\right]$ is a functional of the full propagators and field expectation values of the form :

$$
\begin{aligned}
\beta \Omega & =U(\Phi, \bar{\Phi})+\frac{N}{2} m^{2} v^{2}+N \frac{\lambda}{24} v^{4}-N h v \\
& -(N-1) \frac{i}{2} \int_{k}\left[\ln G_{\pi}^{-1}(k)+D_{\pi}^{-1}(k) G_{\pi}(k)\right] \\
& -\frac{i}{2} \int_{k}\left[\ln G_{\sigma}^{-1}(k)+D_{\sigma}^{-1}(k) G_{\sigma}(k)\right] \\
& +\sqrt{N} i \operatorname{tr}_{D, c} \int_{k}\left[\ln G^{-1}(k)+D^{-1}(k) G(k)\right]+\Gamma_{2 \mathrm{PI}},(7)
\end{aligned}
$$

where the trace is taken in Dirac and color space. The treelevel propagators of the pion, sigma, and constituent quark fields are

$$
i D_{\pi / \sigma}^{-1}(k)=k^{2}-m_{\pi / \sigma 0}^{2}, \quad i D^{-1}(k)=\not k-m_{q},
$$

while $G_{\pi}, G_{\sigma}$, and $G$ are the respective full propagators in terms of which the set of 2PI skeleton diagrams denoted by $\Gamma_{2 \mathrm{PI}} \equiv \Gamma_{2 \mathrm{PI}}\left[G_{\pi}, G_{\sigma}, G, v, \Phi, \bar{\Phi}\right]$ is constructed. To $O(1 / \sqrt{N})$ accuracy this is given by

$$
\begin{aligned}
\Gamma_{2 \mathrm{PI}} & =N \frac{\lambda}{24}\left(\int_{k} G_{\pi}(k)\right)^{2}+\frac{\lambda}{12} \int_{k} G_{\pi}(k) \int_{p} G_{\sigma}(p) \\
& -\frac{\lambda}{12} i \int_{k} \Pi(k)-\frac{i}{2} \int_{k} \ln \left(1-\frac{\lambda}{6} \Pi(k)\right) \\
& -\frac{\lambda}{6} v^{2} \int_{k} G_{\sigma}(k)+\frac{\lambda}{6} v^{2} \int_{k} \frac{G_{\sigma}(k)}{1-\lambda \Pi(k) / 6} \\
& -\sqrt{N} \frac{g^{2}}{2} i \operatorname{tr}_{D, c} \int_{k} \int_{p} \gamma_{5} G(k) \gamma_{5} G(k+p) G_{\pi}(p) \\
& +\frac{g^{2}}{2 \sqrt{N}} i \operatorname{ir}_{D, c} \int_{k} \int_{p} G(k) G(k+p) G_{\sigma}(p),
\end{aligned}
$$

where the notation $\Pi(k)=-i \int_{p} G_{\pi}(p) G_{\pi}(k+p)$ was introduced. The mesonic part of $\Gamma_{2 \mathrm{PI}}$ contains the 2PI diagrams of the $O(N)$ model as given in Eq. (2.13) and Figs. 2 and 4 of [11] and also in Eq. (48) and Fig. 2 of [12]. Notice that the contribution of the fermions goes with fractional powers of $N$ and intercalates between the leading order (LO) and next-to-leading order (NLO) contributions of the pions, which go with integer powers of $N$.

\subsection{The mean-field Polyakov-loop potential}

$U(\Phi, \bar{\Phi})$ appearing in (7) represents one of the different versions of effective Polyakov-loop potentials defined in the literature for $\mu_{q}=0$, where $|\Phi|=|\bar{\Phi}|$. The simplest effective potential is of a Landau type, constructed with terms consistent with the $\mathbb{Z}_{3}$ symmetry [13]:

$\beta^{4} \mathcal{U}_{\text {poly }}(\Phi, \bar{\Phi})=-\frac{b_{2}(T)}{2} \Phi \bar{\Phi}-\frac{b_{3}}{6}\left(\Phi^{3}+\bar{\Phi}^{3}\right)+\frac{b_{4}}{4}(\Phi \bar{\Phi})^{2}$, 
where the temperature-dependent coefficient which makes spontaneous symmetry breaking possible is

$$
b_{2}(T)=a_{0}+a_{1}\left(\frac{T_{0}}{T}\right)+a_{2}\left(\frac{T_{0}}{T}\right)^{2}+a_{3}\left(\frac{T_{0}}{T}\right)^{3} .
$$

$T_{0}$ is the transition temperature of the confinement/deconfinement phase transition, in the pure gauge theory $T_{0}=$ $270 \mathrm{MeV}$. The parameters $a_{i}, i=0, \ldots, 3$ and $b_{3}, b_{4}$ determined in [14] reproduce the data measured in pure $S U(3)$ lattice gauge theory for pressure, and entropy and energy densities. When using this potential in either the PNJL or the PQM models the minimum of the resulting thermodynamic potential is at $\Phi>1$ for $T \rightarrow \infty$, which is not in accordance with the value coming from the definition $\Phi(\mathbf{x})=\left\langle\operatorname{tr}_{c} L(\mathbf{x})\right\rangle / N_{c}$ with $L(\mathbf{x})=\mathcal{P} \exp \left[i \int_{0}^{\beta} d \tau A_{4}(\tau, \mathbf{x})\right]$.

An effective potential for the Polyakov loop inspired by a strong-coupling expansion of the lattice QCD action was derived in [15]. Using the part coming from the $S U(3)$ Haar measure of group integration an effective potential was constructed in [16]

$$
\begin{aligned}
\beta^{4} \mathcal{U}_{\log }(\Phi, \bar{\Phi}) & =-\frac{1}{2} a(T) \Phi \bar{\Phi}+b(T) \ln (1-6 \Phi \bar{\Phi} \\
& \left.+4\left(\Phi^{3}+\bar{\Phi}^{3}\right)-3(\Phi \bar{\Phi})^{2}\right)
\end{aligned}
$$

with the temperature-dependent coefficients

$$
a(T)=a_{0}+a_{1}\left(\frac{T_{0}}{T}\right)+a_{2}\left(\frac{T_{0}}{T}\right)^{2}, \quad b(T)=b_{3}\left(\frac{T_{0}}{T}\right)^{3} .
$$

The parameters $a_{i}, i=0,1,2$ and $b_{3}$ reproduce the thermodynamic quantities in the pure $S U(3)$ gauge theory measured on the lattice. Since the logarithm in $\mathcal{U}_{\log }(\Phi, \bar{\Phi})$ diverges as $\Phi, \bar{\Phi} \rightarrow 1$ the use of this effective potential guarantees that $\Phi, \bar{\Phi} \rightarrow 1$ for $T \rightarrow \infty$.

A third potential is the one determined in Refs. [15]:

$$
\begin{aligned}
\beta \mathcal{U}_{\mathrm{Fuku}}(\Phi, \bar{\Phi}) & =-b\left[54 e^{-a / T} \Phi \bar{\Phi}+\ln (1-6 \Phi \bar{\Phi}\right. \\
& \left.\left.+4\left(\Phi^{3}+\bar{\Phi}^{3}\right)-3(\Phi \bar{\Phi})^{2}\right)\right]
\end{aligned}
$$

where the temperature of the deconfinement phase transition in pure gauge theory is controlled by $a$, while $b$ controls the weight of gluonic effects in the transition. In this case, the parameters $a=664 \mathrm{MeV}$ and $b=(196.2 \mathrm{MeV})^{3}$ are obtained from the requirement of having a first order transition at about $T=270 \mathrm{MeV}[17,2]$.

It was shown in [17] that there is little difference in the pressure calculated from the three effective potentials for the Polyakov loop in their validity region up to $T \simeq$ $(1.5-2) T_{c}$. The presence of dynamical quarks influences an effective treatment based on the Polyakov loop which in this case is not an exact order parameter. Defining effective Polyakov-loop potentials for nonvanishing chemical potential when $|\Phi| \neq|\bar{\Phi}|$ is somewhat ambiguous [2]. Nevertheless, at $\mu_{q} \neq 0$ we use the $\mathbb{Z}_{3}$ symmetric Polyakovloop potentials given above and include the effect of the dynamical quarks along the lines of [2], where using renormalization group arguments the $N_{f}$ and $\mu_{q}$ dependence of the $T_{0}$ parameter of the Polyakov-loop effective potential was parametrized as $T_{0}\left(\mu_{q}, N_{f}\right)=T_{\tau} \exp \left(-1 /\left(\alpha_{0} b\left(\mu_{q}\right)\right)\right)$. The parameters were chosen to have $T_{0}\left(\mu_{q}=0, N_{f}=2\right)=$ 208.64 MeV. When using the Polyakov loop effective potential given in (10) and (12) we will consider in addition to $T_{0}=270 \mathrm{MeV}$ two more cases, one with a constant value $T_{0}=208 \mathrm{MeV}$ and the other with the above-mentioned $\mu_{q}$-dependent $T_{0}$ taken at $N_{f}=2$.

\subsection{The propagator and field equations and the approximations made to solve them}

We are interested in the equations for the two-point functions and the field equations, which are given by the stationary conditions

$$
\frac{\delta \Omega}{\delta G}=\frac{\delta \Omega}{\delta G_{\pi}}=\frac{\delta \Omega}{\delta G_{\sigma}}=\frac{\delta \Omega}{\delta v}=\frac{\delta \Omega}{\delta \Phi}=\frac{\delta \Omega}{\delta \bar{\Phi}}=0 .
$$

In each of these equations the contribution of the fermions is kept only at LO in the large- $N_{f}$ expansion. This contribution is $O(\sqrt{N})$ in the field equations of $\Phi$ and $\bar{\Phi}, O(1)$ in the equation for the fermion propagator $G$, and $O(1 / \sqrt{N})$ in the remaining equations, that is the field equation of $v$, and the equations of $G_{\pi}$ and $G_{\sigma}$.

The second line of (9) does not contribute to any of the equations at the order of interest, and the second term on the right hand side of (9) contributes only in the equation of the sigma propagator

$$
\begin{aligned}
& i G_{\sigma}^{-1}(p)=i D_{\sigma}^{-1}(p)+\frac{\lambda v^{2}}{3}-\frac{\lambda}{6} \int_{k} G_{\pi}(k) \\
& -\frac{\lambda v^{2}}{3} \frac{1}{1-\lambda \Pi(p) / 6}-\frac{i g^{2}}{\sqrt{N}} \operatorname{tr}_{D, c} \int_{k} G(k) G(k+p)
\end{aligned}
$$

In fact, $G_{\sigma}$ will not appear in any of the remaining five equations. Nevertheless, it plays an important role in the parametrization of the model, as discussed in Sec. 2.4.

In the imaginary time formalism of the finite temperature field theory, which we use for calculation, the fourmomentum is $k=\left(i \omega_{n}, \mathbf{k}\right)$, where the Matsubara frequencies are $\omega_{n}=2 \pi n T$ for bosons, while for fermions they depend also on the color due to the color-dependent chemical potential $\mu_{i}$ introduced in (4) and are given by $\omega_{n}=$ $(2 n+1) \pi T-i \mu_{i}$. The meaning of the integration symbol encountered so far is then

$$
\int_{k}=i T \sum_{n} \int_{\mathbf{k}} \equiv i T \sum_{n} \int \frac{d^{3} \mathbf{k}}{(2 \pi)^{3}} .
$$

The dependence on $\Phi$ and $\bar{\Phi}$ of the fermionic trace-log term in the grand potential $\Omega$ and of the quark-pion settingsun in $\Gamma_{2 \mathrm{PI}}$ results from the fact that, after performing the Matsubara sum, the color trace can be expressed in closed form in terms of the mean-field ( $\mathbf{x}$-independent) Polyakov loop $\Phi$ and its conjugate $\bar{\Phi}$. The big difference is that while in case of the trace-log the result can be expressed in terms 
of a modified Fermi-Dirac distribution function

$$
\tilde{f}_{\Phi}^{+}(E)=\frac{\left(\bar{\Phi}+2 \Phi e^{-\beta\left(E-\mu_{q}\right)}\right) e^{-\beta\left(E-\mu_{q}\right)}+e^{-3 \beta\left(E-\mu_{q}\right)}}{1+3\left(\bar{\Phi}+\Phi e^{-\beta\left(E-\mu_{q}\right)}\right) e^{-\beta\left(E-\mu_{q}\right)}+e^{-3 \beta\left(E-\mu_{q}\right)}},
$$

and a similar expression for $\tilde{f}_{\Phi}^{-}(E)$, but with $\Phi \leftrightarrow \bar{\Phi}$ and $\mu_{q} \leftrightarrow-\mu_{q}$, in case of the setting-sun integral the result does not allow for an interpretation in terms of distribution functions, as shown in the Appendix of [7] between Eqs. (A35) and (A37).

Due to the complexity of the problem, the set of coupled equations coming from (15) is only solved using some approximations described below.

1. As a first approximation we disregard the self-consistent equation for the fermions arising from $\delta \Omega / \delta G=0$, that is

$$
i G^{-1}(k)=i D^{-1}(k)-i g^{2} \int_{p} \gamma_{5} G(p) \gamma_{5} G_{\pi}(p-k),
$$

and simply use the tree-level fermion propagator $D(k)$ in the remaining five equations. Within this approximation the field equation of $v$ and the pion propagator simplify considerably. The contribution of the last but one term of (9) to the pion propagator breaks up upon working out the Dirac structure into the linear combination of a fermionic tadpole $\tilde{T}\left(m_{q}\right)$ and a bubble integral $\tilde{I}\left(p ; m_{q}\right)$. Introducing the propagator

$$
D_{0}(k)=\frac{i}{k^{2}-m_{q}^{2}},
$$

these integrals are defined as

$$
\begin{aligned}
\tilde{T}\left(m_{q}\right) & =\frac{1}{N_{c}} \sum_{i=1}^{N_{c}} \int_{k} D_{0}(k), \\
\tilde{I}\left(p ; m_{q}\right) & =\frac{1}{N_{c}} \sum_{i=1}^{N_{c}}\left[-i \int_{q} D_{0}(q) D_{0}(q+p)\right] .
\end{aligned}
$$

In terms of these integrals given between Eqs. (A11) and (A18) of the Appendix of [7], where the sum over the color indices is explicitly done, one obtains

$$
\begin{aligned}
h & \left.=v\left[m^{2}+\frac{\lambda}{6}\left(v^{2}+\int_{k} G_{\pi}(k)\right)-\frac{4 g^{2} N_{c}}{\sqrt{N}} \tilde{T}\left(m_{q}\right)\right],(23)\right) \\
i G_{\pi}^{-1}(k) & =k^{2}-m^{2}-\frac{\lambda}{6}\left(v^{2}+\int_{k} G_{\pi}(k)\right)+\frac{4 g^{2} N_{c}}{\sqrt{N}} \tilde{T}\left(m_{q}\right) \\
& -\frac{2 g^{2} N_{c}}{\sqrt{N}} k^{2} \tilde{I}\left(p ; m_{q}\right) .
\end{aligned}
$$

By making use of the field equation for $v$ in the equation for the pion propagator one finds that $i G_{\pi}^{-1}(k=0)=-h / v$, which means that the Goldstone theorem is fulfilled. Unfortunately, it turns out that this is only accidental, because the Ward identity relating the inverse fermion propagator and the proper vertex $\Gamma_{\pi^{a} \psi \bar{\psi}}=\delta^{3} \Gamma / \delta \bar{\psi} \delta \psi \delta \pi^{a}$ (see $e . g$. Eq. (13.102) of [18])

$$
-\frac{i}{2} T_{a}\left\{\gamma_{5}, i G^{-1}(p)\right\}=v \sqrt{\frac{N}{2 N_{f}}} \Gamma_{\pi^{a} \psi \bar{\psi}}(0, p,-p),
$$

is satisfied only with tree-level propagators and vertices. The relation above is violated at any order of the perturbation theory in the large- $N_{f}$ approximation, for in view of (19) the corrections to the inverse tree-level fermion propagator are of $O(1)$, while the corrections to the tree-level $\pi-\psi-\bar{\psi}$ vertex are suppressed by $1 / N$. The feature is probably a shortcoming of our way of implementing the large- $N_{f}$ scaling in (1), and we could not find a way to overcome it.

2. A further approximation concerns the self-consistent pion propagator (24). In [7] several approximations for $G_{\pi}$ were discussed, here we consider only two of them.

In the chiral limit a local approximation is obtained by parametrizing the pion propagator as

$$
G_{\pi, l}(p)=\frac{i}{p^{2}-M^{2}}
$$

which is then used in all of the equations. $M^{2}$ is determined from $M^{2}=-i G_{\pi, l}^{-1}(p=0)$, which gives the gap-equation

$$
M^{2}=m^{2}+\frac{\lambda}{6}\left(v^{2}+T_{F}(M)\right)-\frac{4 g^{2} N_{c}}{\sqrt{N}} \tilde{T}_{F}\left(m_{q}\right) .
$$

The subscript $F$ denotes the finite part of the cutoff regularized integrals defined in Eqs. (21) and (22). In view of the field equation (23) in the chiral limit $h=0$, one has $M^{2}=0$. We note that due to their self-consistent nature, when (27) is solved, a series containing all orders of $1 / \sqrt{N}$ is in fact resummed.

In the case of a physical pion mass, in addition to the local approximation (26) and (27) for the pion propagator, a nonlocal approximation is derived using an expansion to $O(1 / \sqrt{N})$ in the expression of the pion propagator (24) obtained after exploiting the field equation of $v(23)$ :

$$
G_{\pi}(p)=\frac{i}{p^{2}-\frac{h}{v}}+\frac{2 g^{2} N_{c}}{\sqrt{N}} \frac{i p^{2} \tilde{I}_{F}\left(p ; m_{q}\right)}{\left(p^{2}-\frac{h}{v}\right)^{2}}+O\left(\frac{1}{N}\right)
$$

With this form of the pion propagator the field equation for $v$ reads

$$
\begin{array}{r}
m^{2}+\frac{\lambda}{6}\left(v^{2}+T_{F}(M)\right)+\frac{2 g^{2} N_{c}}{\sqrt{N}} J_{F}\left(M, m_{q}\right) \\
-\frac{4 g^{2} N_{c}}{\sqrt{N}} \tilde{T}_{F}\left(m_{q}\right)=\frac{h}{v}
\end{array}
$$

where in this case $M^{2}=h / v$ and we have introduced the integral

$$
J\left(M, m_{q}\right)=-i \int_{p} G_{\pi, l}^{2}(p) p^{2} \tilde{I}_{F}\left(p ; m_{q}\right) .
$$

Solving this equation for $v$ shows that this approximation also resums infinitely many orders in $1 / \sqrt{N}$.

Before proceeding we note that, since our approximation is not self-consistent, we do not attempt to do a proper renormalization by constructing the counterterms which absorb the divergences of the integrals. It was shown in [7] 
that even when a strict expansion in $1 / \sqrt{N}$ is performed in the pion propagator equation, due to the fact that the fermion propagator is unresummed, different subseries of the counterterms are needed to cancel the subdivergences of different equations. Here we retain the finite parts of the integrals obtained using cutoff regularization. We refer the interested reader to [7] for details.

\subsection{Parametrization}

We need to determine the mass parameter $m^{2}$, the couplings $g, \lambda$ of the Lagrangian (1), the renormalization scale $M_{0 B}$, the vacuum expectation value $v_{0} \equiv v\left(T=0, \mu_{q}=0\right)$, and the external field $h$, which vanishes in the chiral limit. This is done at $T=\mu_{q}=0$ using in addition to the pion decay constant $f_{\pi}=93 \mathrm{MeV}$, pion mass $m_{\pi}=140 \mathrm{MeV}$, and the constituent quark mass taken to be $M_{q}=m_{N} / 3=$ $313 \mathrm{MeV}$ some information coming from the sigma sector, such as the mass and the width of the sigma particle and the behavior of the spectral function.

$v_{0}$ is determined from the matrix element of the axial vector current between the vacuum state and a one-pion state, which due to the rescaling of the vacuum expectation value by $\sqrt{N}$ gives $v_{0}=f_{\pi} / 2$ for $N_{f}=2$. The value of the Yukawa coupling $g=6.7$ is obtained by equating the treelevel fermion mass $m_{q}=g v_{0}$ with $M_{q}$. The parameters $\lambda$ and $M_{0 B}$ are determined from the sigma propagator, as will be detailed below. Having determined them, in the chiral limit $m^{2}$ is fixed from the field equation of $v$, and in the case of the physical pion mass $m^{2}$ is determined from the gap equation by requiring $M^{2}=m_{\pi}^{2}$, and $h$ is obtained from the field equation for $v$, when the local approximation of the pion propagator is used, while when the approximation (28) for $G_{\pi}$ is used, $h$ is fixed by requiring $h=m_{\pi}^{2} v_{0}$, and $m^{2}$ is determined from the field equation of $v$.

In order to fix $\lambda$ and $M_{0 B}$, one uses in (16) the tree-level fermion propagator, the field equation for $v(23)$, and the local approximation (26) for the pion propagator, which means that in the case of a physical pion mass we neglect for simplicity the second term of (29). After working out the Dirac structure one obtains the following form for the sigma propagator:

$$
\begin{aligned}
i G_{\sigma}^{-1}(p) & =p^{2}-\frac{h}{v}-\frac{\lambda v^{2}}{3} \frac{1}{1-\lambda I_{F}(p ; M) / 6} \\
& +\frac{2 g^{2} N_{c}}{\sqrt{N}}\left(4 m_{q}^{2}-p^{2}\right) \tilde{I}_{F}\left(p ; m_{q}\right) .
\end{aligned}
$$

The integral $I_{F}(p ; M)$, obtained using the local approximation (26) for the pion propagator with $M^{2}=m_{\pi}^{2}$, can be found in Eqs. (10) and (11) of [19] with $M_{0}$ replaced by $M_{0 B}$, while $\tilde{I}_{F}\left(p ; m_{q}\right)$ is given in Eqs. (A16)-(A18) of [7].

The self-energy has both in the chiral limit $M=0$ and for $M=m_{\pi}$ two cuts along the positive real axis of the complex $p_{0}$ plane. These are above the thresholds of the pion and fermion bubble integrals, which start at $p^{2}=4 M^{2}$ and $p^{2}=4 m_{q}^{2}$, respectively. Above these thresholds the respective pion and fermion bubble integrals have nonvanishing imaginary parts. We search for poles of the sigma

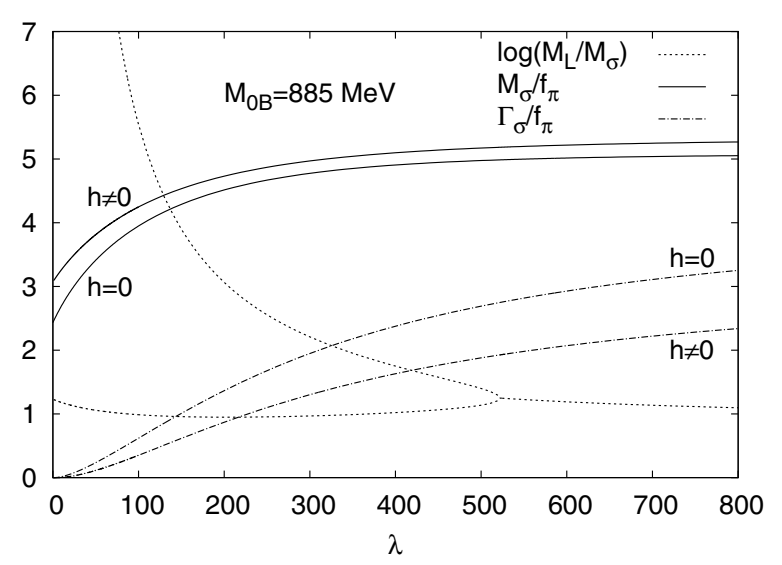

Fig. 1. The $\lambda$ dependence of the real and imaginary parts of the complex sigma pole $p_{0}=M_{\sigma}-i \Gamma_{\sigma} / 2$ and of the Landau ghost $M_{L}$ in the chiral limit and for the physical pion mass indicated with label $h \neq 0$ on the curves. $M_{L}$ is shown only in this latter case, for in the chiral limit there is very little difference.

propagator analytically continued between the two cuts to the second Riemann sheet in the form $i G_{\sigma}^{-1}\left(p_{0}=\kappa e^{-i \phi}, \mathbf{p}=\right.$ $0)=0$. The pole is parametrized as $p_{0}=M_{\sigma}-i \Gamma_{\sigma} / 2$, with the real and imaginary parts corresponding to the mass and the half-width of the sigma particle.

The solution for $M_{\sigma}$ and $\Gamma_{\sigma}$ is shown in Fig. 1 both in the chiral limit $\left(h=m_{\pi}=0\right)$ and for the $h \neq 0$ case. Similar to the case of the $O(N)$ model studied in Ref. [19], in the chiral limit the value of $M_{\sigma}$ is a little smaller and the value of $\Gamma_{\sigma}$ larger than in the $h \neq 0$ case. Comparing Fig. 1 with Fig. 2 of Ref. [19] obtained in the $O(N)$ model, that is without fermions, the $M_{\sigma}(\lambda)$ curve moved slightly upward, but the $\Gamma_{\sigma}(\lambda)$ curve moved significantly downward, which means that in the present case the phenomenologically expected value [20] $M_{\sigma} / \Gamma_{\sigma} \sim 1$ cannot be achieved for any value of the coupling $\lambda$. Another difference is that for low values of $\lambda$ there are two poles of $G_{\sigma}$ on the negative imaginary axis in contrast to only one such pole in the $O(N)$ model. These poles approach each other as $\lambda$ increases and after they collide at a given value of $\lambda$ there are two complex poles at higher $\lambda$, one with positive and one with negative real part. The imaginary part of the complex pole having positive real part is shown in Fig. 1 for the renormalization scale $M_{0 B}=885 \mathrm{MeV}$. As explained in the study done in the chiral limit in [8] for lower values of the renormalization scale the scale $M_{L}$ of the lower Landau ghost on the imaginary axis comes even closer to $M_{\sigma}$ and as a result the spectral function of the sigma is heavily distorted. In order to avoid this and based on the ratio of $M_{\sigma} / \Gamma_{\sigma}$ we have chosen $\lambda=400$ and $M_{0 B}=885 \mathrm{MeV}$. For these values $M_{\sigma}=456 \mathrm{MeV}$ and $\Gamma_{\sigma}=221 \mathrm{MeV}$ in the chiral case, while $M_{\sigma}=474 \mathrm{MeV}$ and $\Gamma_{\sigma}=152 \mathrm{MeV}$ for the case of a physical pion mass. 


\section{The $\mu_{q}-T$ Phase diagram}

The thermodynamics is determined by solving the field equations, i.e. (23) and the equations giving the dependence on $T$ and $\mu_{q}$ of the two real mean fields $\Phi$ and $\bar{\Phi}$, which, when the full fermion propagator is replaced by the tree-level one have the form:

$$
\begin{array}{r}
\frac{d U(\Phi, \bar{\Phi})}{d \Phi}-2 N_{c} \sqrt{N} \int_{\mathbf{k}} \frac{k^{2}}{3 E_{k}}\left(\frac{d \tilde{f}_{\Phi}^{+}\left(E_{k}\right)}{d \Phi}+\frac{d \tilde{f}_{\bar{\Phi}}^{-}\left(E_{k}\right)}{d \Phi}\right) \\
+g^{2} \sqrt{N} N_{c}\left[2\left(\tilde{T}_{F}^{0}\left(m_{q}\right)-T_{F}(M)\right) \frac{d \tilde{T}^{\beta}\left(m_{q}\right)}{d \Phi}+\frac{d \tilde{T}_{2}^{\beta, 2}\left(m_{q}\right)}{d \Phi}\right. \\
\left.-M^{2}\left(\frac{d S^{\beta, 1}\left(M, m_{q}\right)}{d \Phi}+\frac{d S^{\beta, 2}\left(M, m_{q}\right)}{d \Phi}\right)\right]=0,
\end{array}
$$

where $E_{k}=\left(\mathbf{k}^{2}+m_{q}^{2}\right)^{\frac{1}{2}}$ and $M$ satisfies the gap equation (27) or the relation $M^{2}=h / v$. The other equation is similar to (32), the only difference is that the derivative is taken with respect to $\bar{\Phi}$. The integral in (32) is the contribution of the fermionic trace-log integral, while the term proportional with $g^{2}$ is the contribution of the quark-pion twoloop integral in (9) given in Eq. (A35) of [7]. This term is disregarded for simplicity when solving the field equations for $\Phi$ and $\bar{\Phi}$, and only in one case (see the last row of Table 2) the complete equation (32) is solved in order to estimate the error made by neglecting it in all the other cases.

The tricritical point (TCP) and the critical end point (CEP) are identified as the points along the chiral phase transition line of the $\mu_{q}-T$ phase diagram where a 1 st order phase transition turns with decreasing $\mu_{q}$ into a 2 nd order or crossover transition, respectively. In case of a crossover, the temperature $T_{\chi}$ of the chiral transition is defined as the value where the derivative $d v / d T$ has a minimum (inflection point of $v(T)$ ), while the temperature $T_{d}$ of the deconfinement transition is obtained as the location of the maximum in $d \Phi / d T$. The transition point in the case of a 1 st order phase transition is estimated by the inflection point located between the turning points of the multivalued curve $v\left(\mu_{q}\right)$ obtained for a given constant temperature. Although the precise definition of the 1st order transition point is given by that value of the intensive parameter for which the two minima of the effective potential are degenerate, we adopt the definition based on the inflection point because we compute only the derivatives of the effective potential with respect to the fields and propagators.

\subsection{Phase transition in the chiral limit}

In the chiral limit we solve the field equation (23) using the local approximation to the pion propagator (26) with $M^{2}=0$ and neglect the term proportional with $g^{2}$ in (32). The critical temperature of the chiral transition $T_{\chi}$ and the pseudocritical temperature $T_{d}$ of the deconfinement transition at vanishing chemical potential, and the location of the TCP are summarized in Table 1 for various forms of the

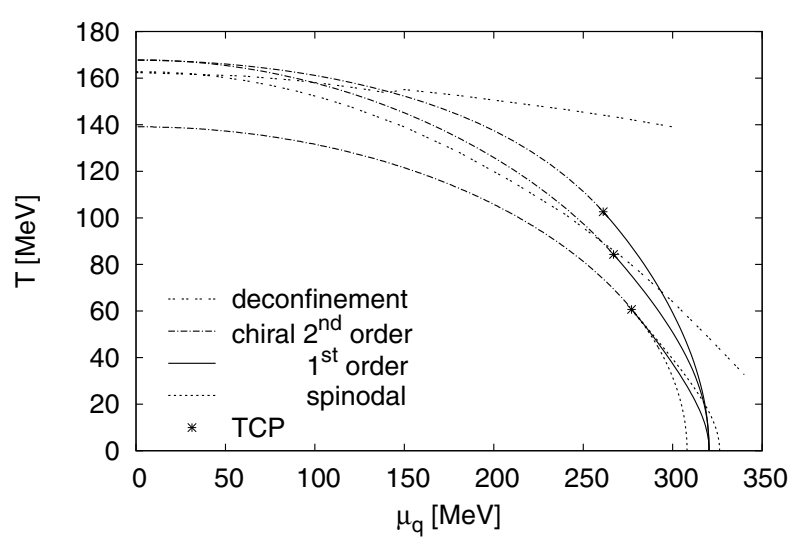

Fig. 2. Phase diagrams obtained in the chiral limit without and with the inclusion of the Polyakov loop. The former has lower $T_{\mathrm{TCP}}$ and for the latter we used $U_{\log }(\Phi, \bar{\Phi})$ with $T_{0}=208 \mathrm{MeV}$ (upper curves) and with $T_{0}\left(\mu_{q}\right)$ (middle curves). The deconfinement transition line is obtained from the inflection point of $\Phi(T)$.

Polyakov-loop potential. With the inclusion of the Polyakov loop $T_{\chi}\left(\mu_{q}=0\right)$ and $T_{\mathrm{TCP}}$ increase significantly compared with the values obtained earlier in [8] without the Polyakov loop, but it has little effect on the value of $\mu_{q}^{\mathrm{TCP}}$. This increase in the value of $T_{\chi}\left(\mu_{q}=0\right)$ is basically determined by the value of the parameter $T_{0}$ of the Polyakov loop potential, while the value of $T_{\mathrm{TCP}}$ shows no significant variation among different cases having the same value of $T_{0}$. One can also see, that as explained in [17], the use of the polynomial and logarithmic effective potentials for the Polyakov loop, that is (10) and (12), drags the value of $T_{\chi}\left(\mu_{q}=0\right)$ closer to the value of the parameter $T_{0}$ than the use of $U_{\text {Fuku }}(\Phi, \bar{\Phi})$ given in (14). In this latter case one obtains the smallest value for $T_{\mathrm{TCP}}$.

For $T_{0}=270 \mathrm{MeV}$ the deconfinement transition line in the $\mu_{q}-T$ phase diagram is above the chiral transition line in all three variants of the effective potential for the Polyakov loop. When the logarithmic effective potential $U_{\log }(\Phi, \bar{\Phi})$ is used either with a constant $T_{0}=208 \mathrm{MeV}$ or with the $\mu_{q}$-dependent $T_{0}$ proposed in [2] one finds $T_{d}<$ $T_{\chi}$ at $\mu_{q}=0$, but at a given value of the chemical potential the deconfinement transition line crosses the chiral transi-

\begin{tabular}{c|c||c|c|c}
\hline$U(\Phi, \Phi)$ & $T_{0}$ & $T_{\chi}(0)$ & $T_{d}(0)$ & $\left(T, \mu_{q}\right)_{\mathrm{TCP}}$ \\
\hline \hline- & - & 139.0 & - & $(60.7,277.0)$ \\
\hline \hline poly & 270 & 185.6 & 229.0 & $(104.5,261.8)$ \\
\hline poly & 208 & 168.2 & 176.5 & $(96.2,263.4)$ \\
\hline $\log$ & 270 & 191.4 & 209.0 & $(109.4,261.2)$ \\
\hline $\log$ & 208 & 167.6 & 162.4 & $(102.6,261.2)$ \\
\hline $\log$ & $T_{0}\left(\mu_{q}\right)$ & 167.9 & 162.8 & $(84.3,266.9)$ \\
\hline Fuku & - & 176.5 & 193.0 & $(99.8,262.2)$ \\
\hline
\end{tabular}

Table 1. The (pseudo)critical temperature $\left(T_{d}\right) T_{\chi}$ of the (deconfinement) chiral transition and the at $\mu_{q}=0$, and the location of the TCP in units of MeV obtained in the chiral limit without the Polyakov loop (first row) and with the inclusion of the Polyakov loop using various effective potentials summarized in Sec. 2.2. 
tion line and remains above it for higher values of $\mu_{q}$. This is shown in Fig. 2, where the deconfinement transition line is obtained from the inflection point of $\Phi(T)$. In contrast to the case of constant $T_{0}$, where basically the deconfinement transition line is not affected by the increase of $\mu_{q}$, with a $\mu_{q}$-dependent $T_{0}$ the deconfinement transition line strongly bends, staying close to the chiral line. The two lines cross just above the TCP.

In the case when $T_{0}\left(\mu_{q}\right)$ is used, the lowering of the deconfinement transition results in the shrinking of the region of the $\mu_{q}-T$ plane for which $T_{\chi}<T<T_{d}$, already observed in Ref. [21]. Since the quantity measuring the quark content inside thermally excited particles carrying baryon number shows a pronounced change along the chiral phase transition line of this region, the region was identified in [17] with the so-called quarkyonic phase, a confining state made of quarks and is characterized by a high quark number density and baryonic (three-quark state) thermal excitations.

Comparing our results on the phase diagram to those obtained in the chiral limit of the PNJL model one can notice differences of both qualitative and quantitative nature. In the nonlocal PNJL model of Ref. [22] the deconfinement phase transition line starts at $\mu_{q}=0$ below the chiral transition line both for a polynomial and a logarithmic Polyakovloop effective potential with $T_{0}=270 \mathrm{MeV}$, so that the two transition lines cross at finite $\mu_{q}$. In our case this happens only for the logarithmic potential with $T_{0}=208 \mathrm{MeV}$, as can be seen in Fig. 2. In [22,23] the values of $T_{\chi}\left(\mu_{q}=0\right)$ and $T_{\mathrm{TCP}}$ are much larger than in our case, while the value of $\mu_{q}^{\mathrm{TCP}}$ is similar to ours.

\subsection{Phase transition with a physical pion mass}

In the approximation (28) for the pion propagator, which resum infinitely many orders in $1 / \sqrt{N}$, the phase transition at $T=0$ turns with increasing $\mu_{q}$ from a crossover type into a first order transition at some value $\mu_{q}^{c}>M_{q}$, and in consequence there is a CEP in the $\mu_{q}-T$ phase diagram. The numerical results are summarized in Table 2 for

\begin{tabular}{c|c||c|c|c|c}
\hline$U(\Phi, \bar{\Phi})$ & $T_{0}$ & $T_{\chi}(0)$ & $T_{d}(0)$ & $\Gamma_{\chi}$ & $\left(T, \mu_{q}\right)_{\mathrm{CEP}}$ \\
\hline \hline- & - & 158.6 & - & 40.7 & $(13.5,328.6)$ \\
\hline \hline poly & 270 & 212.5 & 217.4 & 28.3 & $(32.9,328.8)$ \\
\hline poly & 208 & 184.6 & 176.8 & 22.3 & $(30.6,328.8)$ \\
\hline $\log$ & 270 & 209.7 & 209.3 & 12.0 & $(34.5,329.0)$ \\
\hline $\log$ & 208 & 168.5 & 167.1 & $* 43.0$ & $(33.0,328.9)$ \\
\hline Fuku & - & 195.2 & 191.3 & 21.2 & $(31.8,328.8)$ \\
\hline \hline poly & 208 & 188.1 & 183.1 & 21.4 & $(32.2,329.0)$ \\
\hline
\end{tabular}

Table 2. The temperatures $T_{\chi}$ and $T_{d}$ of the chiral and deconfinement transitions, the half-width at half maximum $\Gamma_{\chi}$ of $-d v / d T$ at $\mu_{q}=0$ (in the case marked with $*$, due to the asymmetric shape of $-d v / d T$, the full width is given) and the location of the CEP in units of $\mathrm{MeV}$ obtained using (28) for the pion propagator without and with the inclusion of the Polyakov loop. The contribution of the quark-pion setting-sun was kept in (32) only for the result of the last row.

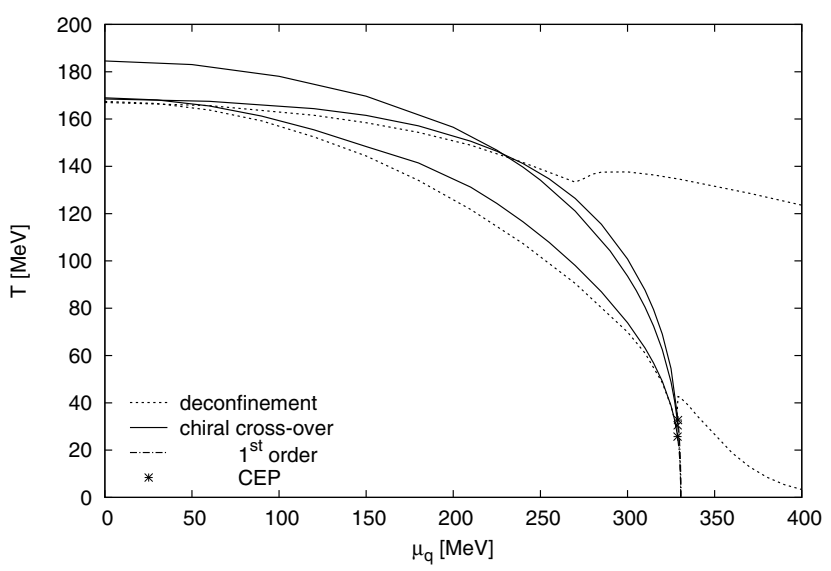

Fig. 3. Phase diagrams obtained for the physical value of the pion mass with the inclusion of the Polyakov loop. For the chiral transition line which starts at higher $T$ for $\mu_{q}=0$ we used $U_{\text {poly }}(\Phi, \bar{\Phi})$ with $T_{0}=208 \mathrm{MeV}$ and (28) for the pion propagator, for the other two phase diagrams we used the local approximation for the pion propagator and $U_{\log }(\Phi, \bar{\Phi})$ with $T_{0}=208 \mathrm{MeV}$ (middle curves) and with $T_{0}\left(\mu_{q}\right)$ (lower curves). The deconfinement transition line is obtained from the inflection point of $\Phi(T)$.

various forms of the Polyakov-loop potential reviewed in Sec. 2.2. Increasing the temperature $\mu_{q}^{c}$ decreases and the first order chiral restoration becomes a crossover at a much lower temperature $T_{\mathrm{CEP}}$ than in the chiral case. The inclusion of the Polyakov loop increases significantly the value of $T_{\mathrm{CEP}}$, but, as in the chiral case, it has little effect on the value of $\mu_{q}^{\mathrm{CEP}}$. Neither the choice of the effective potential for the Polyakov loop nor the value of $T_{0}$ has a significant effect on the value of $\mu_{q}^{\mathrm{CEP}}$. The result in the last row was obtained by keeping in the field equation of the Polyakov loop (32) and its conjugate the contribution of the quarkpion setting-sun diagram, while in all other cases only the contribution of the fermionic trace-log was kept. Comparing the result in the last row of Table 2 with that of the second row obtained using the polynomial Polyakov-loop potential, one sees that the error we make by neglecting the setting-sun contribution in all other cases is fairly small.

The values of $T_{\chi}$ and $T_{d}$ at $\mu_{q}=0$ are mostly influenced by the choice of the Polyakov effective potential and the value of $T_{0}:$ they decrease with the decrease of $T_{0}$ and by using the logarithmic potential instead of the polynomial one. Using the polynomial potential with $T_{0}=270 \mathrm{MeV}$ the confinement transition line in the $\mu_{q}-T$ plane is above the chiral transition line. As in the chiral case, when a logarithmic potential is used with either a fixed value $T_{0}=208 \mathrm{MeV}$ or with a $\mu_{q}$-dependent $T_{0}$, the deconfinement transition line starts at $\mu_{q}=0$ below the chiral one and the two lines cross at some higher value of $\mu_{q}$. This can be seen in Fig. 3. When $T_{0}\left(\mu_{q}\right)$ is used the two lines go together until they cross each other just above the location of the CEP. This $\mu_{q}$-dependent $T_{0}$ gives the lowest value of $T_{\mathrm{CEP}}$, similar to the results reported in [24] and [6]. Because of the much lower value of the $T_{\mathrm{CEP}}$ the shrinking of the quarkyonic phase is more pronounced than in the chiral case, as the deconfinement transition lines ap- 
proaches the $\mu_{q}$ axis. This is even more the case here, with a physical pion mass, since the deconfinement transition is a crossover and as such it happens in a relatively large temperature interval. However, the quarkyonic phase does not vanish completely as happens in [6], where quantum fluctuations are included using functional renormalization group methods.

\section{Conclusions}

Using the tree-level fermion propagator and some approximations for the self-consistent pion propagator obtained within a large- $N_{f}$ expansion, we studied in the $S U(2)_{L} \times$ $S U(2)_{R}$ chiral quark-meson model, in the chiral limit and for the physical value of the pion mass, the influence of the Polyakov loop on the chiral phase transition. When the local part of the approximate pion propagator resums infinitely many orders in $1 / N_{f}$ of fermionic contributions it is possible to find a CEP on the chiral phase transition line of the $\mu_{q}-T$ phase diagram. The inclusion of the Polyakov loop potential has a significant effect on $T_{\text {CEP }}$ and practically no effect on $\mu_{q}^{\mathrm{CEP}}$ obtained in the original chiral quark-meson model, that is which does not contain the Polyakov loop. Using the logarithmic form $U_{\log }(\Phi, \bar{\Phi})$ of the effective potential for the Polyakov loop with parameter $T_{0}=208 \mathrm{MeV}$ a crossing between the chiral and deconfinement transition lines was observed, with the latter line starting at $\mu_{q}=0$ slightly below the former one. In this case the existence of the quarkyonic phase is possible.

It was shown in [7] that the result of resumming in the pion propagator $O(1 / \sqrt{N})$ fermionic fluctuations obtained with a strict expansion in $1 / \sqrt{N}$, while keeping the fermion propagator unresummed, the phase transition softens to the point that there is no CEP in the $\mu_{q}-T$ phase diagram within a range $0<\mu_{q}<500 \mathrm{MeV}$. For this reason it is an interesting question to what extent our results on the existence and location of the CEP would be modified by the use of the self-consistent propagator for fermions, and also by considering the more realistic $S U(3)_{L} \times S U(3)_{R}$ chiral quark-meson model.

\section{Acknowledgments}

This work is supported by the Hungarian Research Fund under Contracts No. T068108 and No. K77534.

\section{References}

1. W. Weise, Prog. Theor. Phys. Suppl. 174, 1 (2008).

2. B.-J. Schaefer, J. M. Pawlowski, and J. Wambach, Phys. Rev. D 76, 074023 (2007).

3. B.-J. Schaefer, M. Wagner, and J. Wambach, Phys. Rev. D 81, 074013 (2010).

4. E. Nakano, B.-J. Schaefer, B. Stokic, B. Friman, K. Redlich, Phys. Lett. B682, 401 (2010).

5. V. Skokov, B. Friman, E. Nakano, K. Redlich, B.-J. Schaefer, Phys. Rev. D 82, 034029 (2010).
6. T. Herbst, J. Pawlowski, and B.-J. Schaefer, arXiv:1008.0081 [hep-ph].

7. G. Markó, Zs. Szép, Phys. Rev. D 82, 065021 (2010).

8. A. Jakovác, A. Patkós, Zs. Szép, and P. Szépfalusy, Phys. Lett. B582, 179 (2004).

9. C. P. Korthals Altes, R. D. Pisarski, and A. Sinkovics, Phys. Rev. D 61, 056007 (2000).

10. J. M. Luttinger and J.C. Ward, Phys. Rev. 118, 1417 (1960).

11. D. Dominici and U. Marini Bettolo Marconi, Phys. Lett. B319, 171 (1993).

12. G. Fejős, A. Patkós, and Zs. Szép, Phys. Rev. D 80, 025015 (2009).

13. R. D. Pisarski, Phys. Rev. D 62, 111501(R) (2000).

14. C. Ratti, M. A. Thaler, and W. Weise, Phys. Rev. D 73, 014019 (2006).

15. K. Fukushima, Phys. Lett. B591, 277 (2004).

16. C. Ratti, S. Rößner, M. A. Thaler, and W. Weise, Eur. Phys. J. C 49, 213 (2007).

17. K. Fukushima, Phys. Rev. D 77, 114028 (2008).

18. J. Zinn-Justin, Quantum Field Theory and Critical Phenomena, 4th edition, (Oxford University Press, Oxford, 2002).

19. A. Patkós, Zs. Szép, and P. Szépfalusy, Phys. Lett. B537, 77 (2002).

20. I. Caprini, G. Colangelo, and H. Leutwyler, Phys. Rev. Lett. 96, 132001 (2006).

21. H. Abuki, R. Anglani, R. Gatto, G. Nardulli, and M. Ruggieri, Phys. Rev. D 78, 034034 (2008).

22. C. Sasaki, B. Friman, and K. Redlich, Phys. Rev. D 75, 074013 (2007).

23. P. Costa, C. A. de Sousa, M. C. Ruivo, and H. Hansen, Europhys. Lett. 86, 31001 (2009).

24. M. Ciminale, R. Gatto, N. D. Ippolito, G. Nardulli, and M. Ruggieri, Phys. Rev. D 77, 054023 (2008). 